\title{
Carrier Thermal Conductivity: Analysis and Application to Submicron-Device Simulation
}

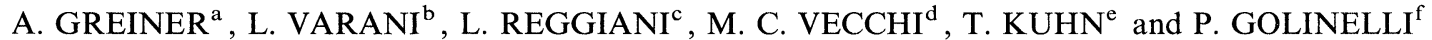 \\ ${ }^{a}$ Institut für Mikrosystemtechnik, Albert-Ludwigs-Universität Freiburg, Am Flughafen 17, 79110 Freiburg, Germany; \\ ${ }^{\mathrm{b}}$ CEM2 (CNRS UMR 5507), Université Montpellier II, 34095 Montpellier Cedex 5, France; \\ ${ }^{\mathrm{c}}$ INFM, Dipartimento di Scienza dei Materiali, Università di Lecce, Via Arnesano, 73100 Lecce, Italy; \\ ${ }^{\mathrm{d}}$ Dipartimento di Elettronica Informatica e Sistemistica, Università di Bologna, viale Risorgimento 2, 40136 Bologna, Italy; \\ ${ }^{\mathrm{e}}$ Institut für Theoretische Physik II, Westfälische Wilhelms-Universität, Wilhelm--Klemm-Str. 10, 48149 Münster, Germany; \\ ${ }^{\mathrm{f}}$ INFM, Dipartimento di Fisica, Università di Modena, Via Campi 213/A, 41100 Modena, Italy
}

\begin{abstract}
Within a correlation-function (CF) formalism, the kinetic coefficientsof charge carriers in semiconductors are studied under different conditions. For the case of linear response in equilibrium, thetransitions from the non-degenerate to the degenerate regimes as wellas from ballistic to diffusive conditions are discussed within ananalytical model. Generalizing the method to high-field transport innondegenerate semiconductors, the CFs are determined by Monte Carlo (MC) calculations for bulk silicon from which the appropriate thermalconductivity has been obtained and included into the hydrodynamic code HEIELDS. For an $n^{+} n n^{+}$submicron structure the temperatureand velocity profiles of the carriers have been calculated with HFIELDS.
\end{abstract}

Keywords: Kinetic coefficients, hydrodynamic transport equations, correlation functions, Monte Carlo

\section{INTRODUCTION}

Thermal conductivity of charge carriers is of fundamental interest in describing transport phenomena in bulk materials as well as electronic devices. To provide a microscopic theory for this coefficient, the $\mathrm{CF}$ approach represents a very effective method [1]. As a consequence of the fluctuation-dissipation theorem, the carrier transport coefficients may be determined by the spectrum of the fluctuations in the system. Results for the thermal conductivity available in literature are mainly based on relaxation-time approximations $[2,3]$. A weighting of the single relaxation time with a power of the energy yields a generalization ofthe Wiedemann-Franz law (WFL) given by

$$
\kappa=\left(\frac{5}{2}+c\right) \frac{k_{B}^{2}}{q} \mu n T,
$$

where $c$ is the so called power law exponent, $\mu$ is the mobility and $T$ the electron temperature. This WFL together with the energy relaxation time is usually introduced within hydrodynamic approaches [4-8].

In this paper we present the appropriate set of $\mathrm{CFs}$ for the cases of linear response around thermodynamic equilibrium in the ballistic and 
diffusive regime under different degeneracy conditions [9], as well as the hot-carrier regime in the classical-diffusive condition $[10,11]$ with its applications to the simulation of an $n^{+} n n^{+}$-structure.

\section{LINEAR RESPONSE REGIME}

In the linear regime, generalized fluxes are the response of the material to generalized externally applied forces mediated by the kinetic (or KelvinOnsager) coefficients $\mathrm{L}_{\mu \nu}$ according to

$$
\mathbf{j}_{\mu}=\sum_{\nu} L_{\mu \nu} \mathbf{X}_{\nu}
$$

with $\mu, \nu=1,2, \mathbf{j}_{1}$ and $\mathbf{j}_{2}$ denoting the electrical current and the energy-flux densities, respectively, while $\mathbf{X}_{1}$ and $\mathbf{X}_{2}$ are the generalized driving forces. The kinetic coefficients $L_{\mu \nu}(\omega)$ in general depend on angular frequency $\omega$ and are defined by means of the Fourier-Laplace transforms $S_{\mu \nu}(\omega)$ of the respective $\mathrm{CFs} C_{\mu \nu}(t)$ from [12]:

$$
L_{\mu \nu}(\omega)=\frac{V S_{\mu \nu}(\omega)}{k_{B} T}
$$

where $V$ is the volume, $k_{B}$ is the Boltzmann constant, $T$ the absolute temperature and:

$$
S_{\mu \nu}(\omega)=\int_{0}^{\infty} d \omega C_{\mu \nu}(t) \exp (-i \omega t) .
$$

Here $C_{\mu \nu}(t)$ is the symmetrized CF at thermal equilibrium

$$
C_{\mu \nu}(t)=\frac{1}{2}\left\langle\delta \mathbf{J}_{\mu}(0) \delta \mathbf{J}_{\nu}(t)+\delta \mathbf{J}_{\nu}(0) \delta \mathbf{J}_{\mu}(t)\right\rangle
$$

where the $\delta \mathbf{J}_{\nu}(t)=\mathbf{J}_{\nu}-\left\langle\mathbf{J}_{\nu}\right\rangle$ denote the operators of the respective observed fluctuating quantities, and $\langle\ldots\rangle$ indicates averaging with respect to the stationary statistical operator. The thermal conductivity $\kappa$ is given as a function of the $L_{\mu \nu}$

$$
\kappa(\omega)=\frac{L_{11}(\omega) L_{22}(\omega)-L_{12}(\omega) L_{21}(\omega)}{T L_{11}(\omega)} .
$$

For a finite one-dimensional conductor of length $\ell$ limited by ideal (i.e. completely absorbing and thermalizing) contacts, if scattering is treated within the relaxation time approximation, the thermal conductivity $\kappa$ can be calculated analytically showing the transitions both from ballistic to diffusive (with increasing $\ell$ ) and from degenerate to non-degenerate (with increasing $T$ ) conditions. The static (i.e. $\omega=0$ ) limiting cases are given by [9]

$$
\begin{gathered}
\kappa^{n d g, b l}=\frac{n k_{B}^{2} T}{m} \tau_{T}^{n d g}=\frac{n \ell k_{B}^{3 / 2} T^{1 / 2}}{(2 \pi m)^{1 / 2}}, \\
\kappa^{d g, b l}=\frac{\pi^{2}}{6} \frac{n k_{B}^{2} T}{m} \tau_{T}^{d g}=\frac{2 \pi^{2}}{3} \frac{\ell k_{B}^{2} T}{h}, \\
\kappa^{n d g, d f}=\frac{3}{2} \frac{n k_{B}^{2} T}{m} \tau_{c}, \\
\kappa^{d g, d f}=\frac{\pi^{2}}{3} \frac{n k_{B}^{2} T}{m} \tau_{c} .
\end{gathered}
$$

Here $\tau_{T}^{n d g / d g}$ is the carrier transit time in the ballistic $(b l)$ case under non-degenerate and degenerate conditions, respectively, while in the diffusive ( $d f$ ) cases $\tau_{c}$ denotes the relaxation time. Figure 1 shows the behavior of $\kappa(0)$ at fixed carrier density as function of $\ell$ and $T$. The maximum value is reached in the ballistic degenerate case.

\section{FAR FROM EQUILIBRIUM (HOT-CARRIER) CASE}

The CFs of microscopic fluxes are calculated with respect to stationary values at the given bias point and the thermodynamic temperature $T$ is replaced by the noise-temperature spectrum $T_{n}(E, \omega=0)$ [13] associated with velocity fluctuations at the given field $E$, where we restrict to the stationary case $(\omega=0)$. The thermal conductivity parallel to the applied electric field, $\kappa(E, \omega=0)$, generalized to hot-carrier conditions is thus given by Eq. (6) with the following replacement:

$$
T \Rightarrow T_{n}(E, \omega)=\frac{V \operatorname{Re}\left[S_{11}(E, \omega)\right]}{k_{B} \operatorname{Re}\left[\sigma_{d}(E, \omega)\right]},
$$




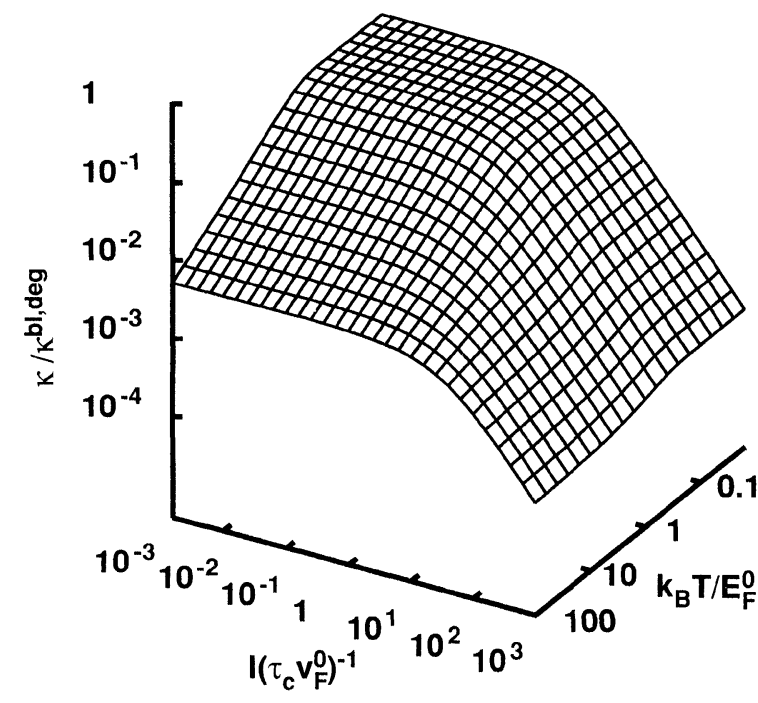

FIGURE 1 Thermal conductivity at zero frequency (normalized to its value in the ballistic degenerate case) at a fixed density $n$ as a function of device length and temperature. $v_{F}^{0}\left(E_{F}{ }^{0}\right)$ denote the Fermi velocity (Fermi energy) at $T=0 \mathrm{~K}$ for the given density $n$ and $\tau_{c}$ is the relaxation time.

where $\sigma_{d}$ is the differential conductivity. Under thermal-equilibrium conditions (i.e. $E=0$ ) $T_{n}(\omega)=T$, and standard linear-response formalism is recovered [14]. The CFs and conductivities entering the definition of $\kappa$ are calculated using MC simulations [15]. We have considered the case of extrinsic $n$-Si, doping concentration $10^{17} \mathrm{~cm}^{-3}$, at $T=300 \mathrm{~K}$. Figure 2 from a) to d) reports the four longitudinal CFs $C_{\mu \nu}(t)$ (fluctuations of flux variable along the electric field direction) at increasing values ofthe electric field normalized to their respective initial values. At increasing fields the correlations decay faster. $C_{22}(t)$ shows the fastest decay, which indicates that its decayrate is the sum of momentum and energy rates. Thus we see that even at thermal equilibrium an approach based on a single time-scale is very poor. We observe that the variances $C_{11}(0), C_{22}(0)$ and $C_{12}(0)=C_{21}(0)$ increase systematically with increasing electric field,which is due to hot-carrier effects. Figure 3 reports thelow frequency spectral densities $S_{\mu \nu}(E, \omega=0)$ of the respective CFs as a function of the electric field. Their different

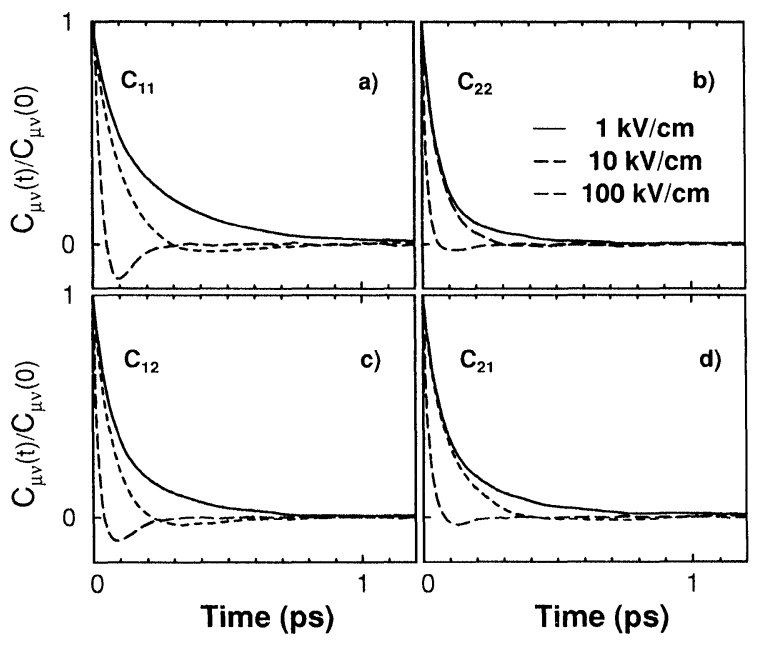

FIGURE 2 Longitudinal CFs normalized to their initial value for extrinsic $n$-Si with doping concentration $n=10^{17} \mathrm{~cm}^{-3}$ at $300 \mathrm{~K}$ and increasing electric-field strengths of 1,10 , and $100 \mathrm{kV} /$ $\mathrm{cm}$.

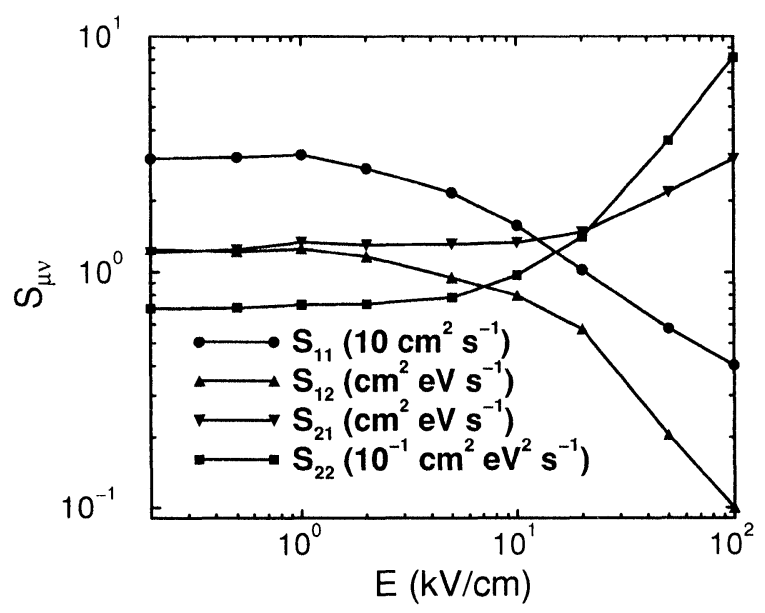

FIGURE 3 Spectral densities of the CFs of Figure 2; at $\omega=0$ as a function of electric-field strength.

behaviors are the result of the competitive effect between shortening time-scales and increasing variances of the corresponding $\mathrm{CFs}$ at increasing fields. The carrier thermal-conductivity at low frequency calculated from Eq. (6) is shown in Figure 4. It is compared to the values obtained from a parameterized WFL (1), for different values of the power law exponent. As can be noticed, the 


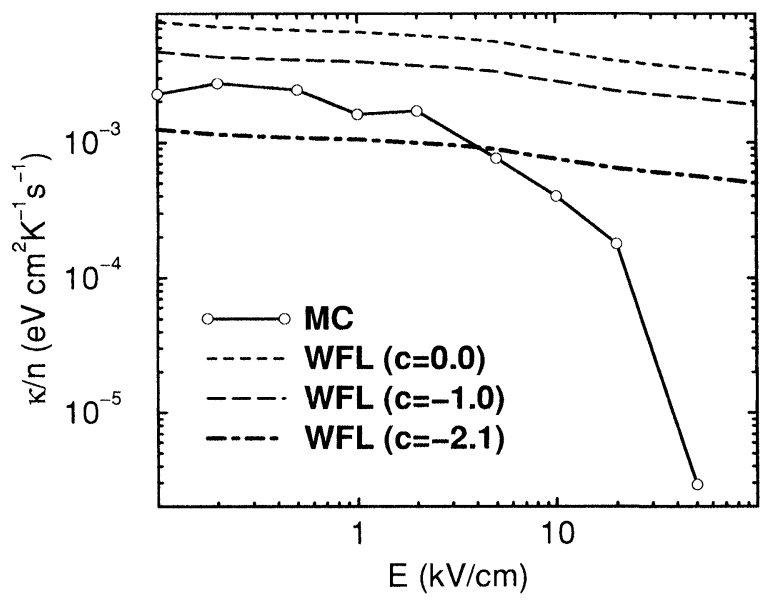

FIGURE 4 Thermal conductivity per unit concentration in $n$ $\mathrm{Si}$ at $300 \mathrm{~K}$ as a function of electric-field strength. The different curves are obtained by setting $c=0.0, c=-1.0$ and $c=-2.1$ in the WFL and by using the CF approach together with MC.

strong decrease at high fields may not be reproduced by a parameterized WFL.

\section{DEVICE SIMULATIONS}

To study the role of thermal conductivity in device csimulation, the transport in a Si submicron $n^{+}-n$ $n^{+}$structure has been analyzed by means of the hydrodynamic device simulator HFIELDS [16]. The simulated structure is the same $n^{+}-n-n^{+}$ diode analyzed in [17]: the high doped $n^{+}$-regions have length $0.1 \mu \mathrm{m}$ and doping concentration $5 \times 10^{17} \mathrm{~cm}^{-3}$, the lower doped $n$-region has length $0.4 \mu \mathrm{m}$ and doping concentration $2 \times 10^{15} \mathrm{~cm}^{-3}$.

The electron mobility $\mu(\varepsilon)$ and energy-relaxation time $\tau_{\varepsilon}(\varepsilon)$ as a function of the mean electron energy $\varepsilon$, required in the hydrodynamic simulator, are calculated from the velocity and energy versus field curves obtained from MC simulations.

For the thermal conductivity both the models obtained from the results of MC simulations and from the parameterized WFL are adopted. In Figure 5 the velocity profile along the device obtained with different models of the thermal conductivity are shown. When the WFL (1) is applied, the spike in the electron velocity curve can be remarkably reduced by setting the value of the power law exponent to $c=-2.1$; at the same time the velocity profiles is smoothed with respect to the MC solution presented in [17] for the same structure. This can be ascribed to the slow decrease of the WFL thermal conductivity with increasing electric field. The $\mathrm{CF}$ approach yields to a stronger dependence of the thermal conductivity on the electric field, which allows to obtain a better comparison with MC data. The carrier temperature profile is shown in Figure 6: alsoin this case the WFL approach tends to smooth the

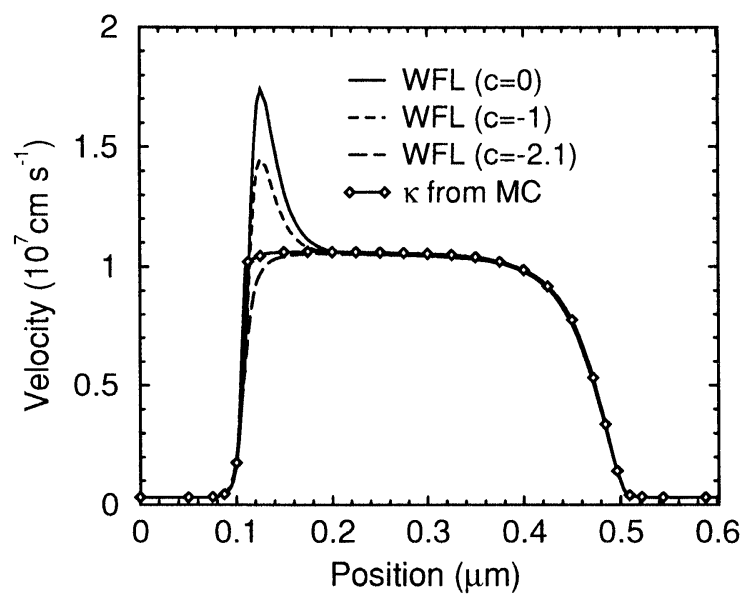

FIGURE 5 Velocity profile of the $n^{+}-n-n^{+}$device calculated with four different thermal conductivities as shown in Figure 4.

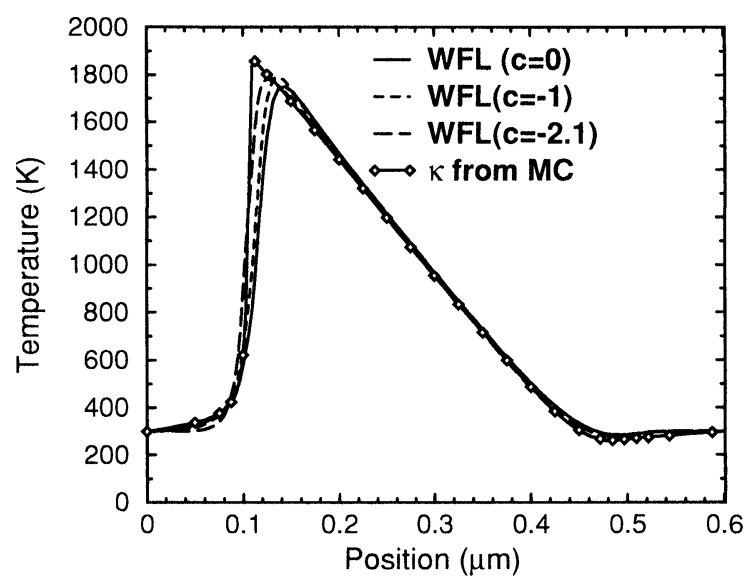

FIGURE 6 Temperature profile of the $n^{+}-n-n^{+}$device for the cases as shown in Figure 5. 
profile with respectto the case when the results of the CF method are applied.

\section{CONCLUSION}

We have shown that the $\mathrm{CF}$ formalism provides an effective method forcalculating electronic transport parameters in different transportregimes. These parameters, calculated in the case of nonequilibriumand incorporated into the hydrodynamic simulatorhfields, allowfor a description of the electronic behavior of submicron structuresin agreement with the results of more sofisticated microscopic approaches.

\section{Acknowledgments}

Support of this work by the Commission of European Community through the contract ERBCHRXCT920047 is gratefully acknowledged. Authors gratefully thank Drs. R. Brunetti and M. Rudan for fruitful discussions.

\section{References}

[1] Kubo, R., Toda, M. and Hashitsume, N. (1991). Statistical Physics II (Springer, Berlin).

[2] Jones, W. and March, N. H. (1973). Theoretical Solid State Physics (Dover New York), 2.

[3] Kireev, P. S. (1974). Semiconductor Physics, 2 ed. (Mir Publishers, Moscow).

[4] Blotekjaer, K. (1970). IEEE Trans. Electron. Devices, ED$17,38$.

[5] Anile, A. and Pennisi, S. (1992). Phys. Rev., B46, 13186.

[6] Stettler, M., Alam, M. and Lundstrom, M. (1993). IEEE Trans. Electron. Devices, ED-40, 733.

[7] Jyegal, J. and Massa, T. D. (1994). Journal of Applied Physics, 76, 4413.

[8] Rudan, M., Vecchi, M. and Ventura, D., In Mathematical problems in semiconductors physics, 340 of Pitman Research Notes in Mathematics Series, edited by, P. Marcati, P. Markowich and R. Natalini (Longman, 1995), pp. 186-214.

[9] Greiner, A., Reggiani, L., Varani, L. and Kuhn, T. (1997). Phys. Rev. Lett., 78, 1114.

[10] Golinelli, P. et al. (1996). In Hot Carriers in Semiconductors, edited by , K. Hess, , J. Leburton and U. Ravaioli (Plenum Press, New York), p. 405.

[11] Golinelli, P., Varani, L. and Reggiani, L. (1996). Phys. Rev. Lett., 77, 1115.
[12] Mohling, F. (1982). Statistical Mechanics (Wiley, New York).

[13] Price, P. (1965). In Fluctuation Phenomena in Solids, edited by, R. Burgess (Academic, New York).

[14] Kubo, R. (1959). In Lectures in Theoretical Physics, edited by, W. Brittin and L. Dunham (Interscience Publs., New York), 1, p. 120.

[15] Brunetti, R. et al. (1981). Journal of Applied Physics, 52, 6713.

[16] Forghieri, A. et al. (1988). IEEE Trans. on CAD of ICAS CAD-7, 231.

[17] Gnudi, A., Odeh, F. and Rudan, M. (1990). European Trans. on Telecommunications and Related Technologies, $\mathbf{1}$ 307.

\section{Authors' Biographies}

Andreas Greiner born 1960 in Ludwigsburg (GERMANY), graduated in physics in 1988 and received the Ph.D. in physics in 1992 from Stuttgart University. His main research activity is in carrier transport simulation of semiconductor devices.

Luca Varani born 1963 in Carpi (ITALY), graduated in physics in 1989, the Ph.D. degree in physics in 1993 from Modena University and inelectronics in 1996 from Montpellier University. $\mathrm{He}$ is researcher at the Centre d'Electronique et de Micro-optoelectronique de Montpellier(CNRS UMR 5507). His main research activity is in the field of electronic transport and noise in semiconductors.

Lino Reggiani, born 1941 in Modena (ITALY), received the Dr. in Physics degree in 1968 and the Diploma di Perfezionamento in Physics in 1972 from Modena University. Since 1994 he is full Professor at the Material Science Department of the Lecce University. His main research activity is in the field of electron transport and fluctuations in semiconductors.

Maria Cristina VECCHI, born 1964 in Avezzano (ITALY), graduated in electrical engineering in 1990 and received the Ph.D. in 1995 from Bologna University. She is a post-doctoral fellow of the DEIS at University of Bologna. Her research activity is in the field of semiconductor device simulations.

Tilmann Kuhn, born 1959 in Alpirsbach (GERMANY), graduated in physics in 1984 and received the Ph.D. in physics from the University 
of Stuttgart. Since 1996 is full professor at the University of Münster. His research interest is in the field of carrier dynamics and fluctuations in semiconductors far from equilibrium on ultrashort length or time scales.
Paola Golinelli, born 1969 in Rivoli (ITALY), received the Dr. in Physics degree in 1995 from Modena University. Her main research activity has been the generalization of charge carrier thermal conductivity to hot-carrier conditions. 

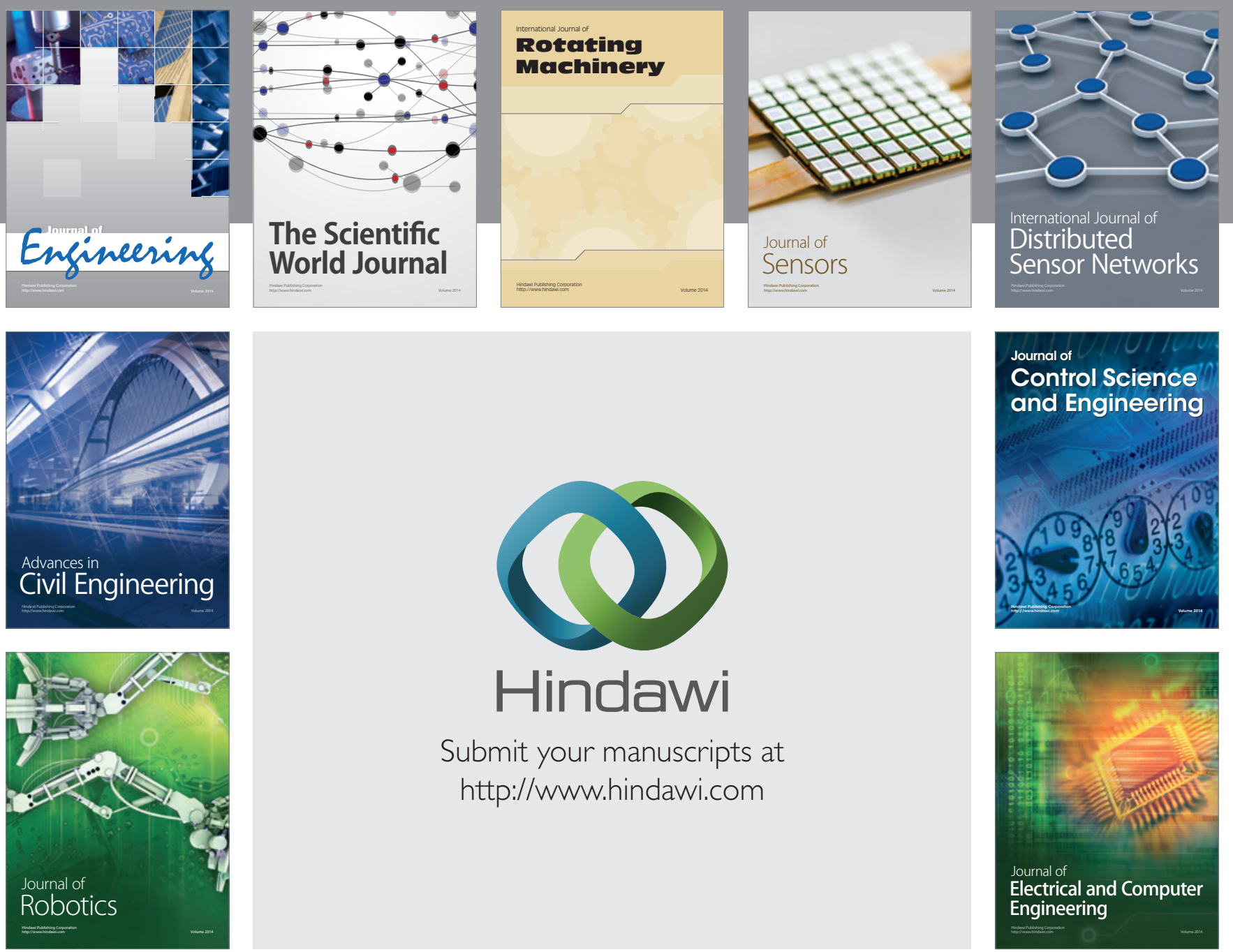

Submit your manuscripts at

http://www.hindawi.com
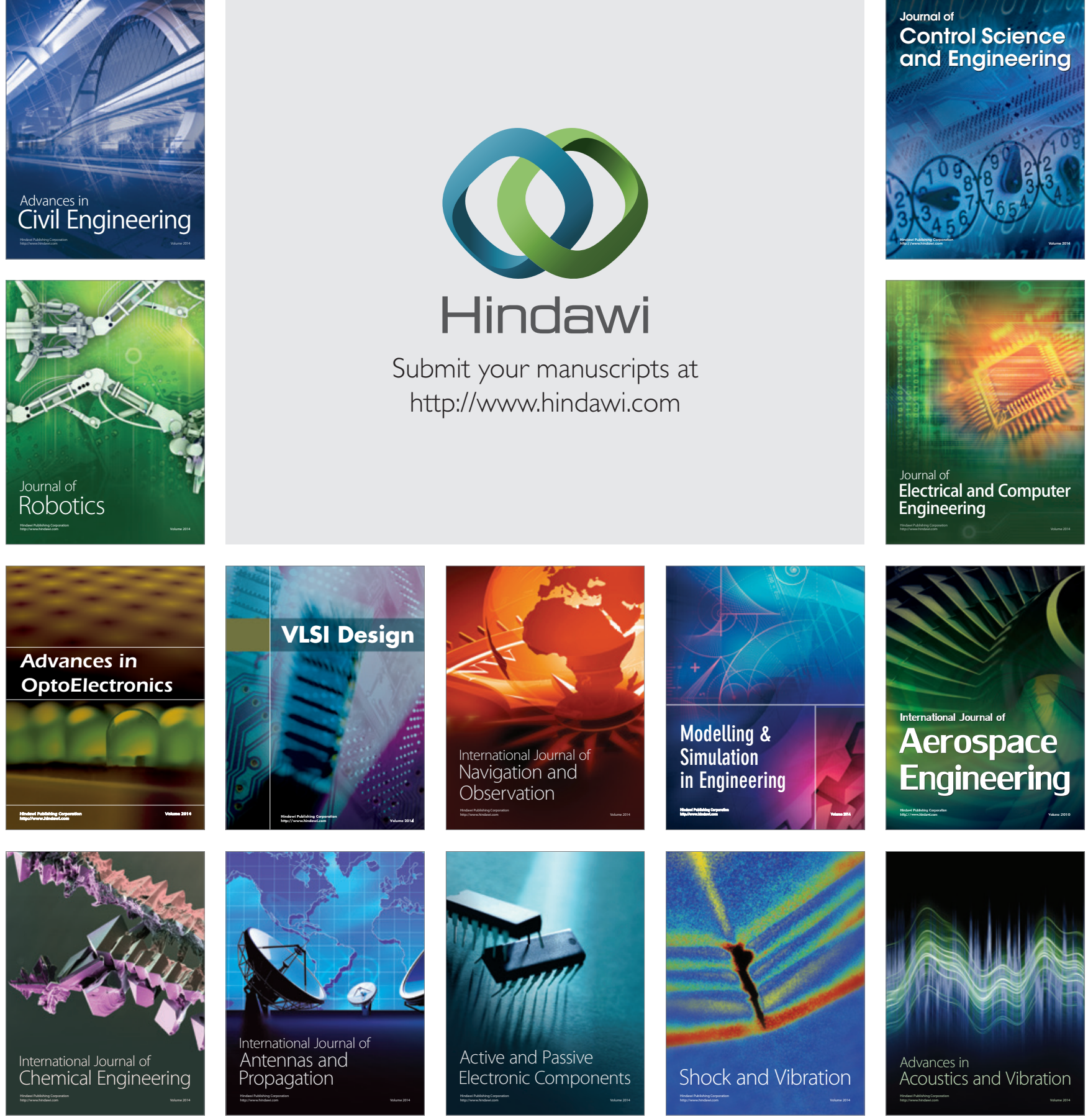